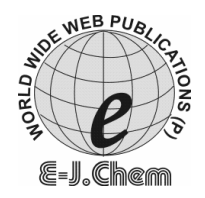

http://www.e-journals.net
ISSN: 0973-4945; CODEN ECJHAO

E-Journal of Chemistry 2009, 6(3), 753-758

\title{
Molecular Mechanics and Quantum Chemistry Based Study of Nickel- $N$-Allyl Urea and $N$-Allyl Thiourea Complexes
}

\author{
P. D. SHARMA, SHRUTI SRIVASTAVA and P. P. SINGH \\ Department of Chemistry, \\ Bareilly College, Bareilly, (U.P.) India. \\ pd_sharma79@rediffmail.com
}

Received 19 October 2008; Revised 2 January 2009; Accepted 15 January 2009

\begin{abstract}
Eigenvalue, eigenvector and overlap matrix of nickel halide complex of $N$-allyl urea and $N$-allyl thiourea have been evaluated. Our results indicate that ligand field parameters (Dq, B' and $\beta$ ) evaluated earlier by electronic spectra are very close to values evaluated with the help of eigenvalues and eigenvectors. Eigenvector analysis and population analysis shows that in bonding $4 s, 4 p$, and $3 \mathrm{~d} x 2-y 2,3 d y z$ orbitals of nickel are involved but the coefficient values differ in different complexes. Out of $4 p x, 4 p y, 4 p z$ the involvement of either $4 p z$ or $4 p y$ is noticeable. The theoretically evaluated positions of infrared bands indicate that $\mathrm{N}$-allyl urea is coordinated to nickel through its oxygen and $\mathrm{N}$-allyl thiourea is coordinated to nickel through its sulphur which is in conformity with the experimental results.
\end{abstract}

Keywords: Eigenvector, Eigenvalues, Population analysis, Spectral parameters.

\section{Introduction}

Nickel(II) halide complexes of $N$-allyl urea (NAU) and $N$-allyl thiourea (NATU) have been studied and their structures have been established with the help of infrared and electronic spectral studies and other physicochemical measurement. These studies have confirmed that both have octahedral structure and the ligand is coordinated to nickel through its oxygen in $\mathrm{NAU}^{1,2}$ and through its sulphur in NATU ${ }^{3-5}$. We have made molecular orbital calculations of the complexes and have evaluated eigenvalue, eigenvector and overlap matrix parameters. The results of these calculations have been correlated with the experimental results. 


\section{Experimental}

The study materials of this paper are $N$-allyl urea and $N$-allyl thiourea complexes of nickel halides, $\mathrm{NiX}_{2} \cdot 4 \mathrm{NAU}$ and $\mathrm{Ni} \mathrm{X}_{2} \cdot 4 \mathrm{NATU}(\mathrm{X}=\mathrm{F}, \mathrm{Cl}, \mathrm{Br}$, I; NAU $=\mathrm{N}$-allyl urea and NATU $=$ $N$-allyl thiourea). The $3 \mathrm{D}$ modeling and geometry optimization ${ }^{6,7}$ of the complexes have been done by CAChe software using molecular mechanics method with EHT option ${ }^{8-11}$.

The electronic spectra excitation energy of the complexes has been evaluated by adopting the same software but by DFT (B3LYP) method ${ }^{12-15}$. Eigenvalues, eigenvectors have also been evaluated by the same software using the EHT option. The method adopted for various calculations is based on the principles described in our earlier paper ${ }^{16}$.

\section{Results and Discussion}

The molecular orbitals of the complex are formed by linear combination of 9 nickel orbitals, 4 orbitals from each halogen and 36 orbitals from each ligand of $\mathrm{NAU}$ in $\mathrm{NiCl}_{2}$.4NAU and 41 orbitals from each ligand of NATU in $\mathrm{NiCl}_{2}$.4NATU complexes.

In total, 161 atomic orbitals are involved in the formation of 161 molecular orbitals in $\mathrm{NiCl}_{2} .4 \mathrm{NAU}$ and 181 atomic orbitals are involved in the formation of 181 molecular orbitals in $\mathrm{NiCl}_{2}$.4NATU. But here we discuss only 17 molecular orbitals of nickel halide, because we have to see the effect of complex formation on these orbitals. Molecular orbitals of ligands are kept out of discussion.

In order to examine the contribution of various atomic orbitals of nickel halides in the formation of molecular orbitals, the LCAO has been studied. The 17 atomic orbitals give LCAO approximation to the 17 molecular orbitals of nickel (II) halide. The various atomic orbitals are represented by $\chi$ and molecular orbitals by $\phi$. The examination of various molecular orbitals $\left(\phi_{1}-\phi_{17}\right)$ and the coefficients of each atomic orbitals $\left(\chi_{1}-\chi_{17}\right)$ reveal interesting results, which are presented below.

$\phi_{1}$ and $\phi_{2}$ have maximum contribution from $n s$ orbitals of the halogens in the complexes $\left(\mathrm{NiX}_{2} .4 \mathrm{NAU}\right)$. The nickel halides $\left(\mathrm{NiX}_{2}\right)$ too have similar contribution from $n s$ orbitals, the eigenvector values or the coefficients of $\chi$ in both the cases are almost the same. The values being $-0.6717,-0.6715$ for $n s$ orbitals of halogen in $\mathrm{NiX}_{2}$ and $0.6173,-0.5656$ for $n s$ orbitals of the halogen in the NAU complexes and -0.6557 , 0.6553 for $n s$ orbitals of the halogen in the NATU complexes. This indicates that extent of involvement of $n s$ orbitals in the formation of molecular orbital in the halides is not altered on complex formation. The molecular orbitals $\left(\phi_{3}-\phi_{4}\right)$ have maximum involvement of $p$ orbitals of the halogens and weak involvement of $d$ orbitals of nickel in both the complexes. The molecular orbitals $\left(\phi_{5}-\phi_{8}\right)$ have maximum involvement of $n p x$, npy and $n p z$ orbitals of the halogens in the halides as well as in their complexes. The coefficient values of $\chi$ however, decreases on complex formation. This decrease is due to the encroachment of the ligands in these orbitals. The extent of encroachment is maximum in sixth molecular orbital followed by seventh. The sequence is as follows:

$$
\begin{array}{ll}
\text { In } \mathrm{NiX}_{2} \text {.4NAU } & 6>7>4>5>8>3 . \\
\text { In } \mathrm{NiX}_{2} \text {.4NATU } & 6>7>4>8>5>3 .
\end{array}
$$

$\phi_{9}$ and $\phi_{10}$ are nonbonding orbitals in nickel chloride. On complex formation, these orbitals do not remain nonbonding. $\phi_{9}$ in the NAU complex are mainly occupied by $3 \mathrm{~d} x y$ and $4 p x$ and in NATU complex by $3 \mathrm{~d} x y$ and $4 p z . \phi_{10}$ in nickel chloride is occupied by $3 \mathrm{~d} x^{2}-y^{2}$ and $3 \mathrm{~d} z^{2}$, which on complex formation is occupied by $3 \mathrm{~d} x^{2}-y^{2}$ and $4 \mathrm{~d} x z$ in NAU complex, 
and by $3 \mathrm{~d} x^{2}-y^{2}$ and $4 p z$ in NATU complex. $\phi_{11}$ is mainly occupied by $3 \mathrm{~d} x y$ and $3 \mathrm{~d} x z$ in both the complexes. The coefficient of $\chi$ decreases from 0.7449 in chloride to $0.6496,0.5134$ in the complexes of NAU and NATU respectively. Molecular orbital $\left(\phi_{12}-\phi_{17}\right)$ are mainly occupied by $n p x, n p y$ and $n p z$ orbitals of halogen in halides whereas in the complex they are occupied by $4 s$ and $4 p x, 4 p y, 4 p z$ orbitals of the nickel. From the observations as discussed above, it is prominently clear that all molecular orbital have contributions from many atomic orbitals.

In order to examine the extent of involvement of $3 d, 4 s$ and $4 p$ orbitals of metal in the formation of molecular orbitals of the complexes the values of coefficients of these orbitals have been separately tabulated for each molecular orbital and are given in Table 1. It shows that there is no nonbonding orbital in complex. All orbitals (five $d$-orbitals, one $s$-orbital and three $p$-orbitals) are taken into consideration. The coefficient value of each orbital has been added to see the total involvement in all the molecular orbitals $\left(\phi_{1}-\phi_{15}\right)$. The summation values are placed at the bottom of the tables. In case of NAU complexes of $\mathrm{NiF}_{2}$ and $\mathrm{NiCl}_{2}$, it is clearly indicated that the maximum involvement is of $d$ orbitals. The involvement of $\mathrm{s}$ and $p$-orbital is almost similar but weak. The value of coefficient of $\mathrm{NiCl}_{2}$.4NAU is between $0.9229-1.9280$ which is very low in comparison to $d$-orbitals $\left(3 d x^{2}-y^{2}, 3 d z^{2}, 3 d x y, 3 d x z, 3 d y z\right)$ which are in the range $2.2129-2.0970$. In case of $\mathrm{NiBr}_{2} .4 \mathrm{NAU}$ and $\mathrm{NiI}_{2} .4 \mathrm{NAU}$, the maximum involvement is of $4 s$ orbitals. The order of involvement of $4 s, 4 p$ and $3 d$-orbital is shown bellow-

$$
4 \mathrm{~s}>3 \mathrm{~d}>4 \mathrm{p} \text {. }
$$

Table 1. Involvement of metal orbitals in bonding, MO No. (1-15).

\begin{tabular}{lccccccccc}
\hline \multicolumn{1}{c}{ Compounds } & $3 d x^{2}-y^{2}$ & $3 d z^{2}$ & $3 d x y$ & $3 d x z$ & $3 d y z$ & $4 s$ & $4 p x$ & $4 p y$ & $4 p z$ \\
\hline $\mathrm{NiF}_{2}$ in $\mathrm{NiF}_{2}$.4NAU & 1.6429 & 0.5671 & 0.4815 & 1.6872 & 1.6387 & 0.6158 & 1.5015 & 1.5173 & 0.9909 \\
$\mathrm{NiCl}_{2}$ in $\mathrm{NiCl}_{2}$.4NAU & 2.2129 & 1.8385 & 2.1906 & 2.097 & 2.1771 & 1.8247 & 1.928 & 1.0015 & 0.9229 \\
$\mathrm{NiBr}_{2}$ in $\mathrm{NiBr}_{2}$.4NAU & 1.4851 & 1.8207 & 1.9542 & 1.4818 & 1.674 & 2.0982 & 0.7849 & 0.8968 & 0.1394 \\
$\mathrm{NiI}_{2}$ in $\mathrm{NiI}_{2}$.4NAU & 0.5988 & 1.4366 & 1.5014 & 1.1343 & 1.2834 & 2.0423 & 1.1303 & 0.932 & 1.9835 \\
$\mathrm{NiF}_{2}$ in $\mathrm{NiF}_{2}$.4NATU & 1.2835 & 0.8813 & 1.0062 & 1.2989 & 1.3377 & 2.6561 & 1.4377 & 1.9434 & 1.1814 \\
$\mathrm{NiCl}_{2}$ in $\mathrm{NiCl}_{2}$.4NATU & 1.7584 & 1.0599 & 1.673 & 1.9075 & 1.8252 & 2.1162 & 1.1996 & 1.3569 & 1.7954 \\
$\mathrm{NiBr}_{2}$ in $\mathrm{NiBr}_{2}$.4NATU & 1.8266 & 1.1867 & 1.1787 & 2.0297 & 2.2483 & 2.0991 & 1.2225 & 1.2182 & 1.6318 \\
$\mathrm{NiI}_{2}$ in $\mathrm{NiI}_{2} .4 \mathrm{NATU}$ & 2.073 & 0.9727 & 1.034 & 1.8332 & 2.03 & 2.1318 & 1.3189 & 1.2579 & 1.5168 \\
\hline
\end{tabular}

In case of $\mathrm{NiX}_{2} .4 \mathrm{NATU}$, it is clearly indicated that the maximum involvement is of $4 \mathrm{~s}$ orbitals. The involvement of $4 \mathrm{p}$ and $3 d$-orbital is almost similar but weak. In case of $\mathrm{NiF}_{2}$.4NATU, the value of coefficient is between $0.8813-1.9434$, which is very low in comparison to $s$-orbital which is 2.6561 .

In case of $\mathrm{NiCl}_{2} .4 \mathrm{NATU}$, the value of coefficient of 4 s-orbital is 2.1162 which is very high in comparison to $4 \mathrm{p}$ and $3 \mathrm{~d}$-orbitals which is in the range 1.0599-1.9075. In case of $\mathrm{NiBr}_{2}$.4NATU, the involvement of $s, p$ and $d$ orbitals are almost the same. The value of coefficient is between 1.1787-2.2483.

In case of $\mathrm{NiI}_{2} .4 \mathrm{NATU}$, the maximum involvement is of $4 s$ orbitals. The involvement of $p$ and $d$-orbital is low. The value of coefficient is between $0.9727-2.0730$ which is very low in comparison to $s$-orbital which is 2.1318 .

The extent of involvement of various orbitals of nickel in the formation of molecular orbitals shows that $s, p$ and $d$ orbitals of nickel have contribution in the formation of molecular orbital in the complexes. 


\section{Population analysis}

The overlap population analysis introduced by Mulliken has also been made for distinguishing bonding, nonbonding and antibonding molecular orbitals. This method allocates proportionally the overlap population $\mathrm{n}_{\mathrm{r}-\mathrm{s}}$ for all possible pairs of basis functions which is shown by the equation- 1 .

where,

$$
\mathrm{n}_{\mathrm{r}-\mathrm{s}, \mathrm{i}}=\mathrm{n}_{\mathrm{i}}\left(2 \mathrm{c}_{\mathrm{ri}} \mathrm{c}_{\mathrm{si}} \mathrm{S}_{\mathrm{rs}}\right)
$$

$\mathrm{c}_{\mathrm{ri}}=$ the coefficient of atomic orbitals for one atom.

$\mathrm{c}_{\mathrm{si}}=$ the coefficient of atomic orbitals for other atom.

and $\mathrm{S}_{\mathrm{rs}}=$ the overlap integral between the two AOs (one of an atom and one of other atom).

The number of electrons has been taken as two for $\phi_{1}-\phi_{15}$. With the help of the eigenvector and overlap integral values, the values of overlap population have been derived from the eq.(1) for the complexes $\mathrm{NiX}_{2} .4 \mathrm{NAU}$ and $\mathrm{NiX}_{2}$.4NATU. The sum of the values of overlap population decides the nature of molecular orbital in a covalent molecule. If the sum is substantially positive the molecular orbital is bonding, if substantially negative the molecular orbital is antibonding and if near zero or zero the molecular orbital is nonbonding ${ }^{17,18}$. The overlap population values shows that first seven molecular orbitals are bonding and the remaining orbitals are antibonding. There are no nonbonding orbitals.

\section{Eigenvalues and orbital splitting}

In case of nickel halide $\left(\mathrm{NiF}_{2}, \mathrm{NiCl}_{2}, \mathrm{NiBr}_{2}, \mathrm{NiI}_{2}\right)$ the eigen values, eigenvector and electronic contribution $\left(\mathrm{n}_{\mathrm{r}, \mathrm{i}}=\mathrm{n}_{\mathrm{i}} \mathrm{c}_{\mathrm{ri}}{ }^{2}\right)$ are same in nonbonding molecular orbitals. The eigen values of the complexes ( $\mathrm{NiX}_{2} .4 \mathrm{NAU}$ and $\left.\mathrm{NiX}_{2} .4 \mathrm{NATU}\right)$ have also been evaluated and are presented in Table 2 . There are no values in these halides which are similar and can support a nonbonding nature of molecular orbitals. This observation further supports the absence of nonbonding orbital in the complexes. The main $d$ orbitals which contribute in the formation of molecular orbital are also shown in the Table 2. The difference in eigen values of $e_{g}$ and $t_{2 g}$ orbitals have been derived in order to obtain theoretical value of $10 \mathrm{Dq}$ in these complexes.

In a text book all $t_{2 g}$ or $e_{g}$ orbitals are shown to be degenerate ${ }^{19}$, but our results indicate that they are not degenerate, and the $\mathrm{d}$ orbitals splitting diagram has to be shown as below.

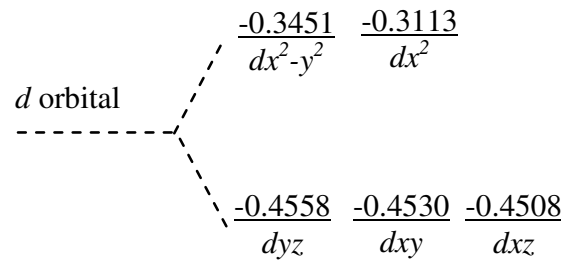

Figure 1. Splitting of $d$-orbitals in $\mathrm{NiCl}_{2}$.4NAU.

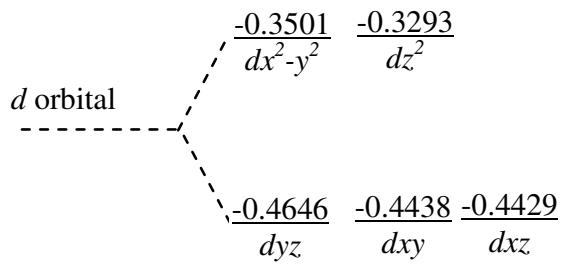

Figure 2. Splitting of $d$-orbitals in $\mathrm{NiBr}_{2}$.4NAU.

The difference in the highest and lowest eigenvalues of the orbitals of $e_{g}$ and $\mathrm{t}_{2 \mathrm{~g}}$ of $\mathrm{NiCl}_{2}$.4NAU and $\mathrm{NiCl}_{2}$.4NATU complex has been chosen for deriving $10 \mathrm{Dq}$ values. The concerned orbitals a re $\mathrm{d} x y$ from $\mathrm{t}_{2 \mathrm{~g}}$ and $\mathrm{dx}^{2}-\mathrm{y}^{2}$ from $e_{g}$ and the corresponding 
eigenvalues are $-0.4558 \mathrm{eV}$ and $-0.3451 \mathrm{eV}$. The difference being $-0.1057 \mathrm{eV}$. This value when converted to $\mathrm{cm}^{-1}$ becomes 852 . Thus, the theoretical $10 \mathrm{Dq}$ value of $\mathrm{NiCl}_{2}$.4NAU complex is $950 \mathrm{~cm}^{-1}$. The experimental value of $\mathrm{Dq}$ is $852 \mathrm{~cm}^{-1}$. On a similar basis the $\mathrm{Dq}$ values in $\mathrm{NiBr}_{2}$. $4 \mathrm{NAU}$ have been evaluated and are presented in Table 2. The calculated $\mathrm{Dq}$ values are close to experimental results and are within the range of Dq prescribed for octahedral structure. The eigenvalues of $t_{2} g$ and $e_{g}$ orbitals of the complexes are presented in Table 2 .

Table 2. Eigenvalues of various $d$ orbitals, $\mathrm{t}_{2 \mathrm{~g}}$ and $e_{g}$

\begin{tabular}{ccccc}
\hline \multirow{2}{*}{ Complexes } & $\mathrm{t}_{2 \mathrm{~g}}$ & $e_{g}$ & \multicolumn{2}{c}{ Da. $\mathrm{cm}^{-1}$} \\
\cline { 3 - 5 } $\mathrm{NiCl}_{\text {.4NAU }}$ & $-0.4558 . d x z$ & $-0.3451 . d z^{2}$ & Calc. & Exp. \\
& $-0.4530, d x y$ & $-0.3113, d x^{2}-y^{2}$ & & \\
& $-0.4508, d y z$ & & \\
$\mathrm{NiBr}_{2}$.4NAU & $-0.4646, d y z$ & $-0.3501, d x^{2}-y^{2}$ & 932 & 916 \\
& $-0.4438, d x z$ & $-0.3293, d z^{2}$ & & \\
& $-0.4429, d x y$ & & & \\
\hline
\end{tabular}

Singh and Pandey ${ }^{1}$ have also evaluated $\mathrm{B}^{\prime}$ and $\beta$ of NAU complexes of nickel(II) halide, with the help of $\mathrm{Dq}$ values and the energies of $v_{2}$ and $v_{3}$ bands. The experimental values obtained by them are presented in Table 3 . Since, we obtained the theoretical values of $\mathrm{Dq}$ with the help of eigenvalues and eigenvector analysis we obtained electronic spectral data for $v_{2}$ and $v_{3}$ from the CAChe software. The values so obtained are listed in Table 3 along with the $\mathrm{B}^{\prime}$ and $\beta$ values evaluated by the secular equation of Tanabe and Sugano ${ }^{20}$. The simplified form of which is given below.

$$
\mathrm{B}^{\prime}=\frac{v_{2}+v_{3}-30 \mathrm{Dq}}{15}
$$

Table 3. Selected bands, assignments and spectral parameters for $\mathrm{NiX}_{2} \cdot 4 \mathrm{NAU}$.

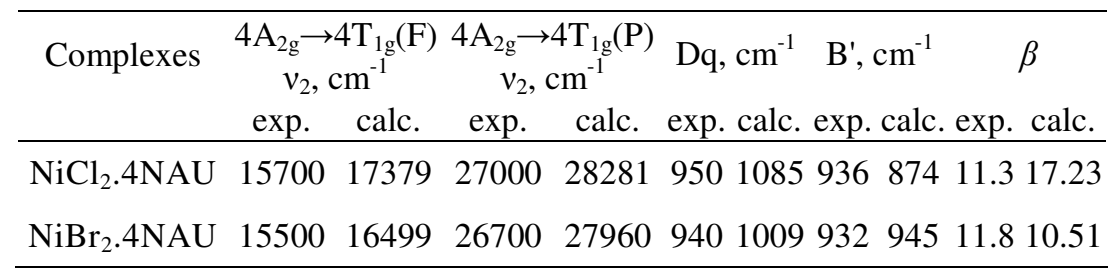

Comparison of the experimental values and the values obtained by theoretical calculation has close resemblance. On detailed examination it is clear that B' values experimentally observed are of the order of $70-90 \%$ of the free ion value $\left(1056 \mathrm{~cm}^{-1}\right)^{20}$, which suggests that there is considerable orbital overlap. This clearly indicates that calculated values are very close to experimental values. The nephelauxetic parameter $\beta$ shows a decrease in the order $\mathrm{Cl}^{-}>\mathrm{Br}^{-}$. The lowest value of $\beta$ for bromide suggests that it has the higher covalency than chloride. Both the experimentally and theoretically obtained values indicate a similar trend.

\section{Conclusion}

The study of eigenvalues, eigenvector and population analysis as presented above have provided valuable information, which is presented below. 
1. The eigenvalue of free nickel ion is $-0.4957 \mathrm{eV}$. In $\mathrm{NiF}_{2}, \mathrm{NiCl}_{2}, \mathrm{NiBr}_{2}$ and $\mathrm{NiI}_{2}$ this energy is retained only in two orbitals which are identified as $\mathrm{d} z^{2}$ and $\mathrm{d} y z$. The population analysis has clearly shown that these orbitals are nonbonding in nature.

2. The population analysis clearly indicated that $9^{\text {th }}$ and $10^{\text {th }}$ orbital of $\mathrm{NiF}_{2}$ and $\mathrm{NiCl}_{2}$ are nonbonding where as in $\mathrm{NiBr}_{2}$ and $\mathrm{NiI}_{2}$ they are $6^{\text {th }}, 7^{\text {th }}$ orbitals.

3. On complex ( $\mathrm{NiX}_{2} .4 \mathrm{NAU}$ and $\left.\mathrm{NiX}_{2} .4 \mathrm{NATU}\right)$ formation, no orbital remains nonbonding in nature.

4. The splitting of $d$ orbital is maximum in case of $\mathrm{NiI}_{2}$ and lowest in $\mathrm{NiF}_{2}$. This shows that orbital overlap is maximum in iodide which is well related with nephelauxetic parameter. On complex formation, the same situation is retained; however, the $\mathrm{Dq}$ values have a different order.

5. The theoretically evaluated values of ligand field parameters such as Dq, B' and $\beta$ are very close to the experimental results. Hence, the theoretical methods based on molecular mechanics calculation can be well relied upon.

6. The eigenvector analysis and population analysis have shown that in bonding only $s$ and $d$ orbitals are involved in halides $\left(\mathrm{NiX}_{2}\right)$ but in complexes $\left(\mathrm{NiX}_{2} .4 \mathrm{NAU}\right.$ and $\mathrm{NiX}_{2}$.4NATU) all $s, p$ and $d$ orbitals are involved.

7. Generally, the crystal field splitting diagram of octahedral complexes are represented by sets of degenerate orbital i.e. $e_{g}$ and $\mathrm{t}_{2 \mathrm{~g}}$. The precise study however shows that the two sets of $d$ orbitals are not degenerated but they slightly differ in their energies.

8. The results of theoretical calculations favor bonding through oxygen in NAU and through sulphur in NATU complexes.

\section{References}

1. Singh P P and Pande I M, Canadian J Chem., 1972, 50, 2603.

2. $\quad$ Singh P. P and Rivest R, Canadian J Chem., 1968, 46, 2361.

3. Scuseria G E, Chem Phys Lett., 1995, 243, 193.

4. Zimmer M, Chem Rev., 1995, 95, 2629.

5. Madan S K and Goldstein C, J Inorg Nucl Chem., 1966, 28, 1251.

6. Madan S K and Mueller D, J Inorg Nucl Chem., 1966, 28, 177.

7. Baker J, Kessi A and Delley B, J Chem Phys., 1996, 105, 192.

8. Lipkowitz K, J Chem Educ., 1995, 72, 1070.

9. Rappe A K and Casewit C J, Molecular Mechanics Across Chemistry, University Science Books, 1997.

10. Landis C R, Lipkowitz K B and Boyd D B Reviews in Computational Chemistry, VCH: 1995, 6, Chapter 2.

11. Madan S K and Sulich M, Inorg Chem., 1966, 5, 1662.

12. Becke A D, Phys Rev., A 1988, 38, 3098.

13. Lee C, Yang W and Parr R G, Phys Rev., B 1988, 37, 785.

14. Jursic B S, Int J Quantum Chem., 1997, 61, 93.

15. Barone V and Adamo C, Int J Quantum Chem., 1997, 61, 443.

16. Singh P P and Sharma P D, Russian J Coordination Chem., 2008, 34(3), 210.

17. Levine I N, Quantum Chemistry, $5^{\text {th }}$ Ed., Prentice Hall, New Jersey, 2000, 506.

18. Bachrach S M, Lipkowitz K and Boyd D B, Reviews in Computational Chemistry, VCH, 1994, 5, Chapter 3.

19. Huheey J E, Keiter E A and Keiter R L, Inorganic Chem, $4^{\text {th }}$ Ed. Pearson Education, 2002, 403, Chapter 11.

20. Tanabe Y and Sugano S, J Phys Soc Jpn., 1954, 9, 753. 


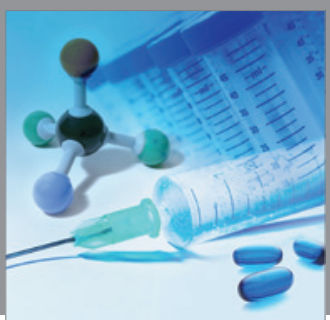

International Journal of

Medicinal Chemistry

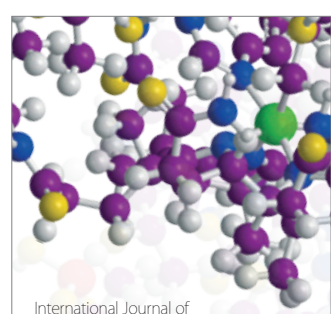

Carbohydrate Chemistry

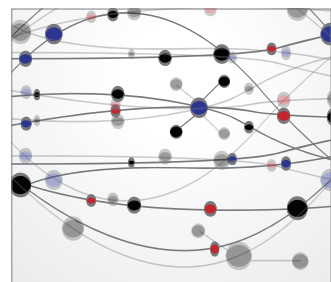

The Scientific World Journal
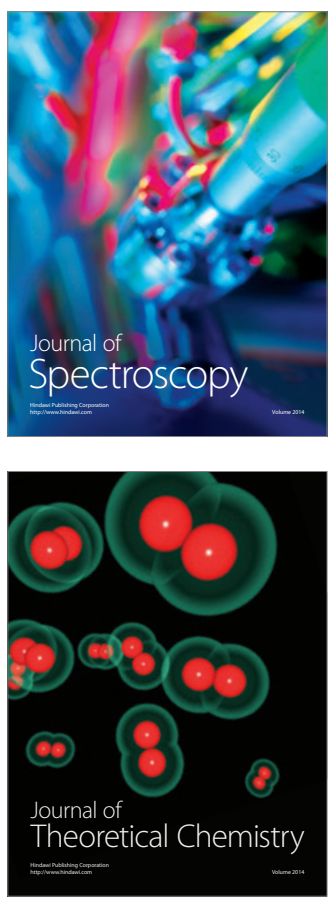
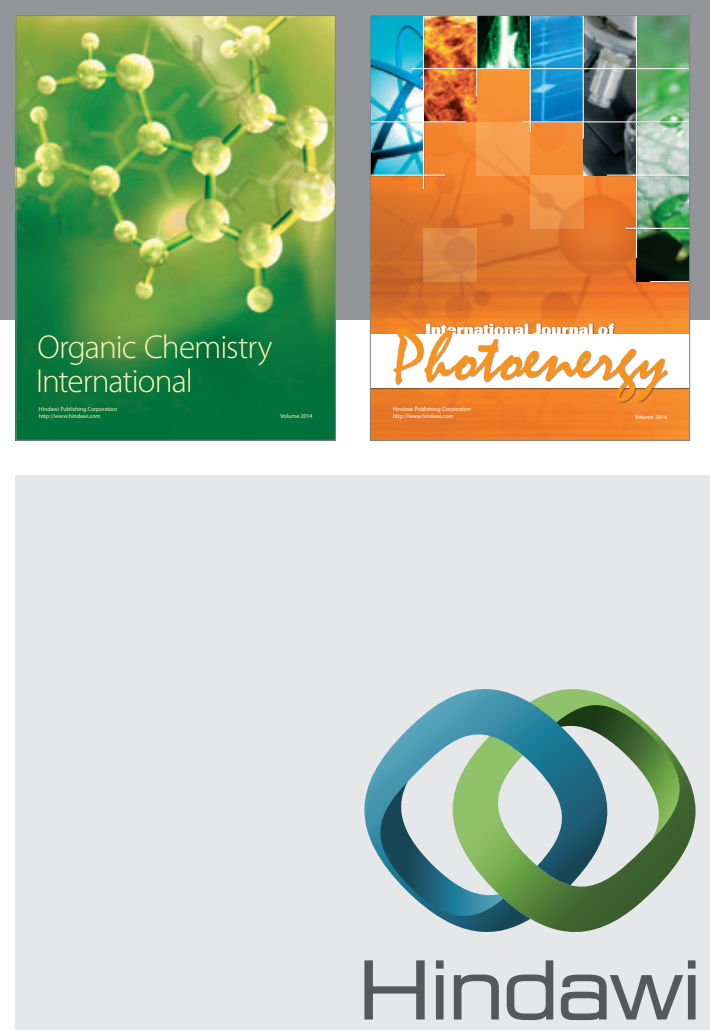

Submit your manuscripts at

http://www.hindawi.com
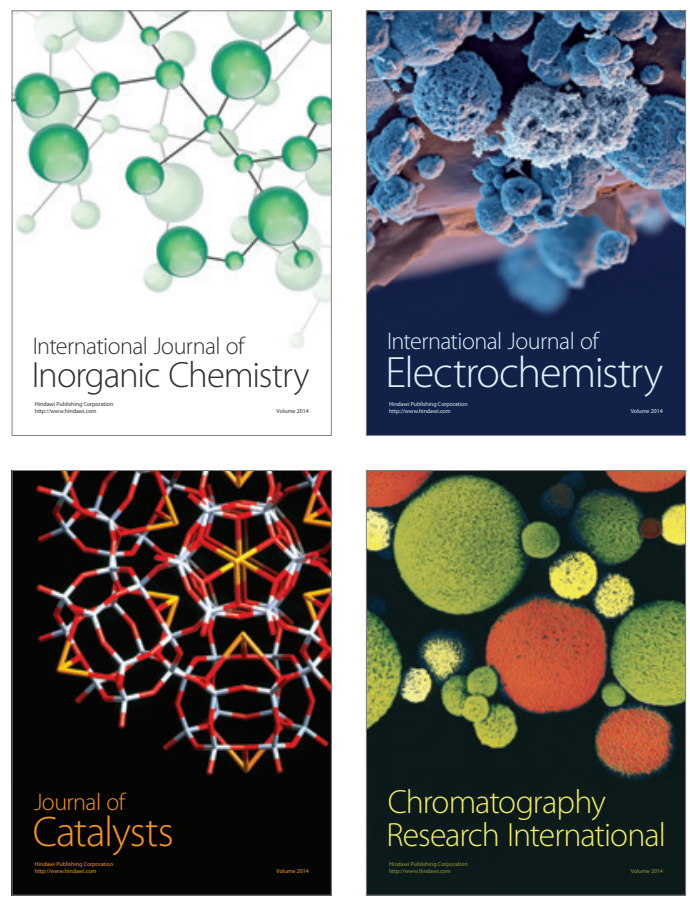
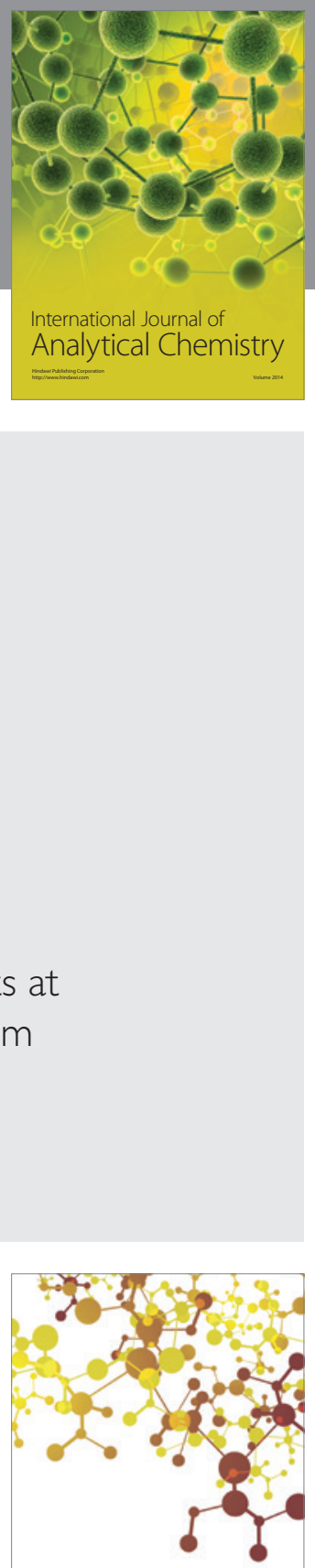

Journal of

Applied Chemistry
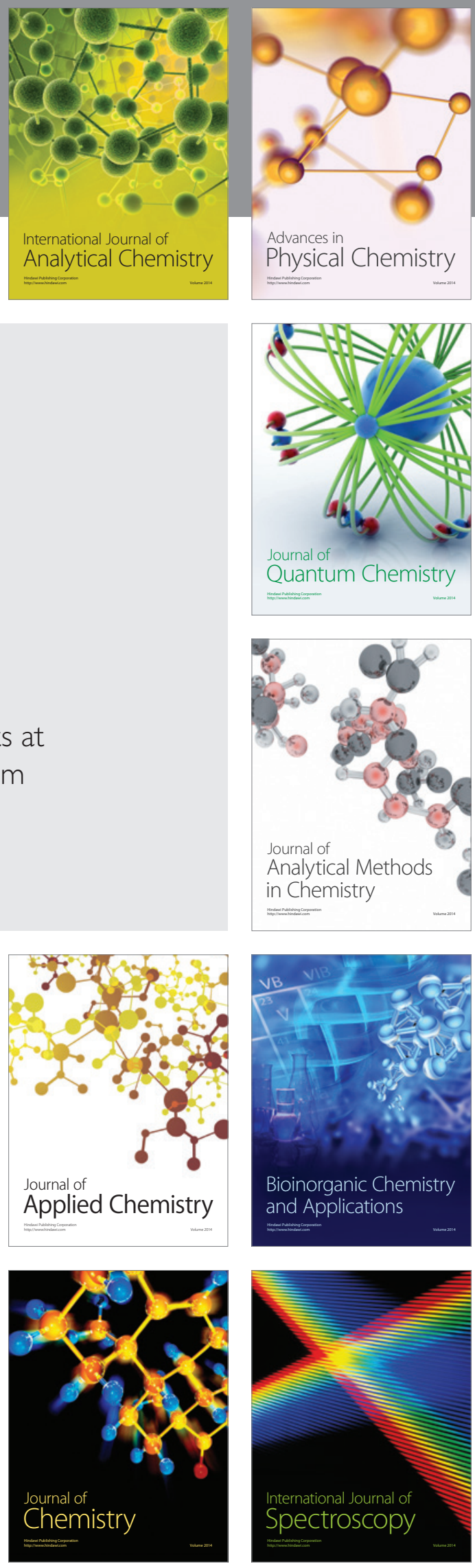